\title{
RÉSULTATS ÉLECTROENCÉPHALOGRAPHIQUES DANS 33 CAS DE TUMEURS INTRACRANIENNES CHIRURGICALEMENT VÉRIFIFES
}

\author{
Paulino W. Loxgo * \\ Pauli) Pinto Pupo** \\ Olavo Pazzantese ***
}

A. Mattos Pimfita $* * * *$

Dès 1914, nous recherchons les possibilités diagnostiques de l'électroencéphalographie dans les tumeurs intracraniennes, au point de vue de l'existence possible d'une tumeur, mais surtout de sa localisation par rapport à la surface extérieure du crâne ${ }^{1}$.

À ce propos nous nous sommes orientés par les travaux de W. G. Walter et collaborateurs ${ }^{2}$, de Case et Bucy ${ }^{3}$, de Williams et Gibbs ${ }^{4}$, de Kornmüller ${ }^{5}$, de Smith, Ch. Walter et Laidlaw ${ }^{6}$, de Foster et Nims ${ }^{7}$, de $\mathrm{Cobb}^{8}$, de Yager et Luse ${ }^{9}$, de Williams et Reynell ${ }^{10}$, de Jasper et Hawke ${ }^{11}$, de Puech et Lerique-Koechlin ${ }^{12}$, de Ulett ${ }^{13}$, de Guerenstein et Strauss ${ }^{14}$, de Glaser et Sjaardema ${ }^{15}$ et, plus récemment, par ceux de Bagchi et Basset ${ }^{16}$.

Méthode - Tous les examens utilisés dans le présent travail on été exécutés avec un appareil de Grass, de six canaux: 12 électrodes ont été mis à la surface du crâne et deux aux oreilles. Plus tard, nous avons utilisé des électrodes medianes et nous avons fait l'exploration monoauriculaire, d'après Bagchi et Basset. Les techniques de dérivation furent: unipolaires avec électrodes indifférentes auriculaires; liaisons en chaines longitudinales, transversales, circulaires et triangulaires, toutes en prise hipolaire visant déterminer les relations de phases. Nous avons toujours préferé d'étu-

Trabalho apresentado ao 4." Congresso Internacional de Neurologia, reunido em Paris, em setembro de 1949.

* Professor de Neurologia da Escola Paulista de Medicina; Diretor do Serviço de Ncuro-Psiquiatria do Instituto Paulista.

* Docente de Neurologia da Fac. Med. da Univ. de São Paulo. Chefe de Clínica Neurológica da Escola Paulista de Medicina.

** Do Instituto de Electrencefalografia (São Paulo).

**** Neuro-cirurgião do Serviço de Neurologia da Fscola Paulista de Medicina. 
dier simultanéinent des points homologues des deux hémisphères, car l'appareil de six canaux est fort usable pour cette recherche.

Matériel - Les malades éxaminés venaient de plusieurs hôpitaux et de la clinique particulière. Les examens ont été faits de préférence avant les autres procédés semiologiques capables d'introduire une possible cause d'erreur dans les données électriques (ponction rachidienne, pneumoencéphalographie et ventriculographie, artériographie, etc.).

Le nombre de patients suspects de tumeur endocranienne fut de 75 , parmi lesquels 33 furent vérifiés par la chirurgie ou la ventriculographie. Parmi les cas non vérifiés chirurgicalement, l'évolution clinique élimina la possibilité de tumeur 11 fois. Dans 4 cas, la neuro-chirurgie montra des lésions non tumorales. Dans 15 cas l'EEG n'accusa pas de tumeur; dans 2 cas à évolution clinique clairement tumorale, l'EEG accusa l'existence de la neoplasie. Il nous a été impossible de suivre l'évolution de 25 cas.

Le présent travail comprend seulement les 33 cas vérifiés par la neurochirurgie ou la ventriculographie. Parmi ces cas, 26 tumeurs étaient localisées dans un des hémisphères cérébrales, 25 ayant donné des signes électroencéphalographiques de latéralisation $(96,1 \%$ des cas $)$.

Par rapport aux types d'altérations localisées dans les cas de tumeurs d'hémisphère, on constate:

\begin{tabular}{|c|c|}
\hline$E E G$ & Nombre de cas \\
\hline Ondes delta $\ldots \ldots \ldots \ldots \ldots \ldots \ldots \ldots$ & 23 \\
\hline Réversion de phases $\ldots \ldots \ldots \ldots \ldots \ldots$ & 19 \\
\hline Dépression du rythme de bases $\ldots \ldots$. & 12 \\
\hline
\end{tabular}

Dans un cas l'EEG ne donna pas de signes de tumeur (patient opéré un mois après, avec glioblastome multiforme). En 2 cas de tumeurs de la base, sans répercussion directe ou indirecte sur le cerveau, l'EEG fui normal. Dans un cas d'abcès du cervelet il $y$ avait répercussion clinique ni électrique sur le cerveau (syndrome d'hypertension endocranienne, signes électriques locaux occipitaux). Dans un cas de gros neurinome de l'acoustique droit, apparut le rythme theta bilatéral avec forte prédominance droite.

Un autre aspect intéressant est la relation entre le type de la tumeur et les altérations de l'EEG. Bien que le nombre de cas ne permette pas des conclusions définitives, on peut observer dans le tableau suivant les données se rapportant à ces éléments: 


\begin{tabular}{|c|c|c|c|c|c|c|}
\hline \multirow{2}{*}{$\begin{array}{l}\text { Trpe de Trmetr } \\
\text { Supra tentorielle: }\end{array}$} & \multirow{2}{*}{$\begin{array}{l}\text { N.9 } \\
\text { de } \\
\text { cas }\end{array}$} & \multicolumn{2}{|c|}{$\begin{array}{c}\text { Rythmes de } \\
\text { base }\end{array}$} & \multicolumn{2}{|c|}{ Altérations } & \multirow{2}{*}{$\begin{array}{l}\text { Repercu- } \\
\text { tions, pour } \\
\text { le cote } \\
\text { oppost }\end{array}$} \\
\hline & & oui & non & diffuses & focales & \\
\hline Gliomes $\quad \ldots \ldots \ldots \ldots \ldots \ldots$ & 15 & 9 & 6 & 8 & 12 & 10 \\
\hline Tumeurs métastiques $\ldots \ldots \ldots$ & 2 & 1 & 1 & 1 & 2 & 2 \\
\hline Vasculaires $\ldots \ldots \ldots \ldots \ldots$ & 3 & 2 & 1 & 1 & 3 & - \\
\hline Meningéomes $\ldots \ldots \ldots \ldots \ldots$ & 4 & 3 & 1 & - & 4 & 2 \\
\hline $\begin{array}{l}\text { Abcès cérébral } \ldots \ldots \ldots \ldots \ldots \\
\text { Kyste comprimant le lobe }\end{array}$ & 1 & 1 & - & -- & 1 & 1 \\
\hline $\begin{array}{l}\text { frontal } \ldots \ldots \ldots \ldots \ldots \ldots \\
\text { Tumeur kystique intra ventri- }\end{array}$ & 1 & 1 & - & - & 1 & - \\
\hline $\begin{array}{c}\text { culaire } \\
\text { Tumeur affectant le diencé- }\end{array}$ & 1 & 1 & - & - & 1 & - \\
\hline phale $\quad \ldots \ldots \ldots \ldots \ldots \ldots$ & 2 & 2 & & Rythme & bilateral & - \\
\hline $\begin{array}{l}\text { Adenome de l'hypophyse } \ldots \ldots \\
\text { Infra tentorielle: }\end{array}$ & 1 & $\begin{array}{l}\text { nor- } \\
\text { mal }\end{array}$ & & & & \\
\hline Abcè & 1 & - & 1 & 1 & 1 & - \\
\hline Neurinomes du acoustique ... & 2 & 2 & - & 1 & $1(\Theta)$ & 1 \\
\hline
\end{tabular}

\section{CONCLUSIONS}

1. L'EEG a donné des signes de latéralisation dans 25 cas de tumeurs de l'hémisphère cérébral $(96 \%$ des cas $)$.

2. Signes de syndrome focal ont été rencontrés dans 25 sur 26 cas de tumeurs localisées aux hémisphères $(89 \%)$.

3. Le syndrome d'hypertension endocranienne, tout en agissant sur l'EEG, n'a pas empêché l'apparition des signes focaux (11 sur 13 cas).

4. Le rythme theta est apparu bilatéralement dans 2 cas de tumeur du diencéphale et dans un cas de gros neurinome de l'acoustique droit, ce dernier avec prédominance dans la région occipital droite.

5. Les anomalies de l'EFG les plus fréquentes furent les ondes delta (26 sur 29 cas) et la dépression ou suppression du rythme alpha (13 sur 29 cas). Dans deux cas, la suppression focale du potentiel fut l'unique anomalie observée. Dans les autres cas, ces altérations sont apparues combinées aux ondes delta.

6. Des tumeurs intracraniennes les gliomes sont ceux qui ont donné le maximum d'altération diffuses. Les meningiomes et les tumeurs vasculaires ont donné des altérations focales.

7. La répercussion contrelatérale est plus fréquente dans les tumeurs intra-cérébrales et profondes; nous l'avons rencontrée dans 2 cas de meningiome, dans un cas de tumeur du $1 / 3$ antérieur de la faux avec envahisse. ment hemisphérique bilatéral et dans un autre cas qui présentait, en plus d'un meningiome, un kyste gliomateux.

8. Un cas de glioma diffus n'a pas donné d'altérations du EFG (l'opération à été faite un mois après l'examen). 


\section{BIBLIOGRAPHIE}

1. a) Pupo, P. P. e Mattos Pimenta, A. - A Eletroencefalografia nos tumores cranianos. Arq. Neuro-Psiquiat., (S. Paulo), 4:373-390 (dezembro) 1946. b) Pupo, P. P., Pazzanese, O. e Mattos Pimenta, A. - A Eletrencefalografia nos tumores intracranianos. Arq. Neuro-Psiquiat. (S. Paulo), 6:1-24 (março) 1948. c) Longo, P. W., Pupo, P. P., Mattos Pimenta, A., Lemmi, O. - Tumor da região do terceiro ventrículo. Diagnóstico electroencefalográfico. Arq. NeuroPsiquiat. (S. Paulo), 6:169-177 (junho) 1948.

2. a) Walter, W. G. - The location of cerebral tumours by electroencephalography. Lancet, 2:305 (agôsto) 1936. b) Walter, W. G. - The technique and applications of electroencephalography. J. Neurol. a. Psychiat., (Londres), $1: 359-85$, 1938. c) Walter, W. G. e Dovey, V. J. - Electroencephalography in cases of subcortical tumour. J. Neurol. Neurosurg. a. Psychiat. (Londres), 7:57-65, 1944.

3. a) Case, Th: J. e Bucy, I. - Localization of cerebral lesions by electroencephalography. J. Neurophysiol., 1:425-261, 1938. b) Case, Th. J. - Electroencephalography in the diagnosis and localization of intracranial lesions. J. Nerv. a. Mental Dis., 87:598, 1938.

4. Williams, D. e Gibbs, F. A. - Electroencephalography in clinical neurology. Its value in routine diagnosis. Arch. Neurol. a. Psychiat., 41:519-534, 1939.

5. Kornmüller, $\Lambda$. E. - Einige weitere Erfahrungen über die Lokalisation von Tumoren und anderen herdförmigen Erkrankungen des Gehirns mittels der hirnbioelektrischen Lokalisationsmethodik. 'Lentralb. f. Neurochir., 5:75-85, 1940.

6. Smith, J. R., Walter, Ch. e Laidlaw, R. - The electroencephalogram in cases of neoplasms of the posterior fossa. Arch. Neurol. a. Psychiat., 43:472-487, 1940.

7. Foster, F. e Nims, K. - Electroencephalographic effects of acute increase of intracranial pressures. Arch. Neurol. a. Psychiat., 47:449-153, 1912.

8. Cobb, W. A. - 'The electroencephalographic localization of intracranial neoplasms. J. Neurol. Neurosurg. a. Psychiat. (Londres), 7:96-102, 1944.

9. Yaeger, Ch. e Luse, S. - Electroencephalogräphic localization and differentiation of lesions of trontal lobes (pathologic confirmation). Arch. Neurol. a. Psychiat., 54:197-201, 1945.

10. Williams, D. e Reynell, J. - Abnormal supression of cortical trequencies. Brain, 48:123-162, 1945.

11. a) Jasper, H. e Hawke, W. - Electroencephalography. IV. Localization of seizure waves in epilepsy. Arch. Neurol. a. Psychiat., 39:885-902, 1938. b) Jasper, H. - The place of electronecephalography in clinical neurology. A restrospective and prospective study. Arch. Neurol. a. Psychiat., 44:1345-1347, 1940 .

12. Puech, M. P. e Lerique-Koechlin, M. - L'électro-encéphalographie dans les abscés du cerveau. Rev. Neurol., 76:303-304, 1944.

13. Ulett, G. - Electroencephalogram of dogs with experinental space-occupying intracranial lesions. A rch. Neurol. a. Psychiat., 54:141-149, 1945.

14. Guerenstein, L. e Strauss, H. - Correlations between electroencephalogram and histologic structure of gliogenous and metastatic brain tumors. J. Mt. Sinai Hosp., 12:874-877, 1945. Resumo in Year-Book Neurol., Psychiat., a. Endocrinol., Chicago, 1945.

15. Glaser, M. A. e Sjaardema, H. - Effect on the electroencephalogram of localized pressure on the brain. J. Neurophysiol., 9:63-72, 1945.

16. Bagchi, B. K. e Basset, R. C. - Some additional electroencephalographic techniques for the localization of intra-cranial lesions. J. Neurosurg., 4:348-369, 1947. 